\title{
Management of HIV in Children Using a Bovine Colostrum-Based Food Product- An Observational Field Study
}

\author{
Patrick Olwedo Odong', Pamela Judith Angwech'², James Obol $^{3}$, Julius Kuule², \\ Claes-Henrik Florén ${ }^{*}$ \\ ${ }^{1}$ Amuru District Local Government, Gulu, Uganda \\ ${ }^{2}$ Gulu Women Economic Development and Globalisation, Gulu, Uganda \\ ${ }^{3}$ Faculty of Medicine, Gulu University, Gulu, Uganda \\ ${ }^{4}$ Department of Medicine, Division of Clinical Sciences, Lund University Hospital, Lund, Sweden \\ Email: ${ }^{*}$ claes-henrik.floren@med.lu.se
}

Received 3 March 2015; accepted 28 May 2015; published 3 June 2015

Copyright (C) 2015 by authors and Scientific Research Publishing Inc.

This work is licensed under the Creative Commons Attribution International License (CC BY). http://creativecommons.org/licenses/by/4.0/

(c) (i) Open Access

\section{Abstract}

Aim: Malnutrition increases the severity and mortality of HIV infection. Therefore, this field study was started and aimed to provide a major cohort of HIV-infected children with the colostrumbased food product ColoPlus (ColoPlus AB, Malmö, Sweden) and to investigate the effects of ColoPlus on the nutritional status and immunological capacity of children including tolerability and safety of the product. Methods: In this major field program comprising 850 malnourished HIV positive children, 50 grams of ColoPlus was administrated for 4 weeks as the first meal in selected health facilities in Northern Uganda. Forty-eight of these children (8 months - 14 years of age) were recruited into a descriptive prospective study and were followed for 12 weeks. At the start (week 0) and at weeks 4,8 and 12, CD4+ cell counts, serum albumin and hemoglobin were analyzed. The remaining 802 patients were observed to register safety and tolerability of ColoPlus. Results: There was a general improvement of wellbeing of the children with increased body weight and decreased fatigue. In the descriptive study of the 48 children, there was a significant rise of the CD4+ values at week $4(+15.4 \% \pm 2.8 \%, p=0.0001)$ compared to week 0 , and at week 8 $(+39.1 \% \pm 3.9 \%, p<0.0001)$, but a return towards the 0 -values at week $12(+2.1 \%, \pm 2.8 \%$, NS $)$. Hemoglobin and serum albumin showed an almost similar trend. ColoPlus was well tolerated by all the 850 children and no side effects or adverse events were seen. Conclusion: These results show that addition of a colostrum-based food product to the daily diet is beneficial in HIV-positive malnourished children. An improvement in nutritional status as well as in immune capacity was seen. These effects were prolonged and remained at least 4 weeks after cessation of ColoPlus administration.

\footnotetext{
${ }^{*}$ Corresponding author.
}

How to cite this paper: Odong, P.O., Angwech, P.J., Obol, J., Kuule, J. and Florén, C.-H. (2015) Management of HIV in Children Using a Bovine Colostrum-Based Food Product-An Observational Field Study. World Journal of AIDS, 5, 100-104. 


\section{Keywords}

\section{CD4+, Bovine Colostrum, Colostrum-Based Food Supplement, HIV Infected Children}

\section{Introduction}

Malnutrition increases severity and mortality of HIV infection. HIV and malnutrition tend to occur in the same population, the underprivileged and resource-poor. Uganda has striven to fight the HIV pandemic with a multisectorial approach which has led to a drop in the national sero-prevalence from $18 \%$ in the early 1990 's to a sero-prevalence now of $7.3 \%$.

Colostrum is the first milk the lactating cow gives to the calf. Bovine colostrum is a very rich source of fat, protein, sugar and micronutrients. Bovine colostrum has also very high levels of several bioactive components such as antimicrobial peptides (e.g. lactoferrin) and immunoglobulins (IgG, IgM, IgA) active against a wide range of bacterial, viral and protozoal pathogens as well as against toxins [1]-[6].

The biological function of colostrum is, through its composition, to provide the calf with passive immunity until its own intestinal immune system is fully functional, and thereby help to prevent infections resulting in diarrhea. Another function is to counteract malnutrition and support growth of the calf through its high levels of nutrients and growth factors.

ColoPlus (ColoPlus AB, Malmö, Sweden) is a bovine colostrum-based food product consisting of two components, one bioactive part (bovine colostrum) and one food product vehicle. The vehicle is composed of food product particulate matter, which is used to slow down time required for emptying the stomach and to slow down small intestinal transit time, thus prolonging intestinal exposure to colostrum. ColoPlus comes as a dry powder and has to be mixed with lukewarm water to obtain a porridge suitable for consumption [7] [8].

\section{Aim of the Study}

Earlier studies in adult patients have shown that ColoPlus provides good nutritional support and boosts the CD 4+ counts [7] [8]. ColoPlus has also been shown to be well tolerated and without any side effects in adults [7] [8]. This study, being the first in children, was aimed at investigating the effects of ColoPlus on the nutritional status and immunological capacity in a large cohort of HIV infected children.

\section{Methods}

\subsection{Study Setting}

The study was conducted in the three districts of Gulu, Amuru and Nwoya in Northern Uganda. Children were from community health facilities of Kaladima health centre III, Anaka Hospital and Lalogi health centre IV.

\subsection{Study Patients}

Study patients were recruited, examined and followed up at their different sites in the health facilities. The inclusion criteria were HIV/AIDS positive children 8 months to 14 years old, who had the following; malnutrition $\leq-3 \mathrm{Z}$ score to $\leq-2 \mathrm{Z}$ score, on cotrimoxazole prophylaxis, but not on anti-retroviral (ARV) therapy or on tuberculosis treatment. Exclusion criteria were children who were known to be lactose intolerant and who were taking immune booster supplements.

\subsection{Study Design}

This was a major prospective cohort study in which 850 malnourished HIV-positive children, aged eight months to fourteen years, were given a total of $50 \mathrm{~g}$ of ColoPlus daily for four weeks as either the first meal in the morning or as repeated daily portions.

Separately, forty-eight of these children, who were also HIV-positive (22 females and 26 males, 8 months to 14 years of age, mean age 6.4 years) were recruited into a descriptive prospective study and followed for up to 
12 weeks (Table 1). Follow up outcomes at the start (week 0 ) and at weeks 4, 8 and 12 were: body weight, fatigue, CD4+ cell count, serum albumin and hemoglobin. The laboratory tests were done at Joint Clinical Research Centre of Excellence in Gulu, Uganda. As the measured blood sample values and body weight differed widely between individual patients (Table 1), each patient served as his own control, and differences at 4, 8 and 12 weeks were calculated percentagewise using each patient's baseline value (at week 0 ) as reference value (Table 2).

Fatigue level was estimated by the patient using a visual analogue scale (VAS) consisting of a line drawn with equal interval scale from 0 to 10 where 0 represents no fatigue and 10 represents the worst fatigue level possible.

The remaining 802 patients were enrolled for observation and monitored through interviews and focus group discussions to observe effects, and to register tolerability and safety of ColoPlus. The interviews were structured in two ways depending on the age of the study participants. On one hand one-to-one interviews with children 8 years and older were conducted while on the other hand interviews with parents to children under 8 years were held. In this group of 802 children ColoPlus effects were expressed as estimates and no quantifiable data on a structured basis were obtained.

\subsection{Statistical Analysis}

A data base was developed and data was entered directly into the computer using Excel software. Mean values and standard deviations were calculated. Comparisons of values before and after treatment with ColoPlus were done with Student's t-test.

\subsection{Ethical Consideration}

Ethical approval was obtained from Institutional Review Board of Gulu University, Uganda and from Uganda National Council for Science and Technology (UNCST) and clearance was also obtained from Uganda National Drug Authority (NDA) for the use of ColoPlus product in Uganda. Informed consent was obtained from the study participants and parents after properly explaining to them, in the language they understood (the Luo language), the purpose, procedures, benefits and potential risks of participating in the study. They indicated consent by endorsing pre-designed consent forms using their signatures or thumbprints in the presence of the investigator or research assistants. The patients further had the right to refuse to participate or withdraw from the study at any time if they so wished. Their confidentiality and privacy was highly observed.

Table 1. Baseline characteristics of the study group.

\begin{tabular}{|c|c|c|c|}
\hline Number of children & \multicolumn{3}{|c|}{48} \\
\hline Gender & \multicolumn{3}{|c|}{$\begin{array}{c}\text { Females } 22(46 \%) \\
\text { Males } 26(54 \%)\end{array}$} \\
\hline Age & \multicolumn{3}{|c|}{ Mean 6.4 years (range 8 months to 14 years) } \\
\hline Malnutrition status & \multicolumn{3}{|c|}{$\leq-3 \mathrm{Z}$ score to $\leq-2 \mathrm{Z}$ score } \\
\hline Hemoglobin levels & \multicolumn{3}{|c|}{ mean $11.0 \mathrm{~g} / \mathrm{L}$ (patients range from 8.8 to 13.3 ) } \\
\hline Serum albumin levels & \multicolumn{3}{|c|}{ mean 38.7 g/L (patients range from 29.7 to 46.6) } \\
\hline CD4+ cell counts & \multicolumn{3}{|c|}{ mean 842 cells $/ \mu L$ (patients range from 32 to 2896) } \\
\hline & 4 weeks & 8 weeks & 12 weeks \\
\hline CD4+ counts & $\begin{array}{c}+15.4 \% \pm 2.8 \% \\
p=0.0001\end{array}$ & $\begin{array}{c}+39.1 \% \pm 3.9 \% \\
p<0.0001\end{array}$ & $\begin{array}{c}+2.1 \% \pm 2.8 \% \\
\text { NS }\end{array}$ \\
\hline Hemoglobin & $\begin{array}{c}+6.7 \% \pm 0.14 \% \\
p=0.0014\end{array}$ & $\begin{array}{c}+12.0 \% \pm 0.09 \% \\
\mathrm{p}<0.0001\end{array}$ & $\begin{array}{c}+5.2 \% \pm 0.15 \% \\
p=0.0196\end{array}$ \\
\hline S-albumin & $\begin{array}{c}+7.4 \% \pm 0.11 \% \\
p<0.0001\end{array}$ & $\begin{array}{c}+12.0 \% \pm 0.09 \% \\
\mathrm{p}<0.0001\end{array}$ & $\begin{array}{c}+5.2 \% \pm 0.15 \% \\
p=0.0196\end{array}$ \\
\hline
\end{tabular}




\section{Results}

All the 850 participants showed very good acceptability to ColoPlus. None of the participants showed any side effects or intolerance to ColoPlus.

Out of 802 children in the observational group, around 200 had severe diarrhea, around 150 had mild diarrhea and around 350 were having a normal stool frequency at the start of the study. A great number of children who had diarrhea reported complete cessation of diarrhea in the second week after commencing consumption of ColoPlus. However, these data were collected by focus group discussions and key informant interviews, so the exact figures cannot be given. There was, though, a considerable effect of ColoPlus in reduction and control of diarrhea. There was also reported weight increases and decrease in fatigue.

The sub-group of 48 children, of which 22 were females and 26 were males, were followed separately and blood samples were collected. In all these children there was a considerable increase in general well-being and a decrease in fatigue as measured by a visual analogue scale. At the beginning of the study the visual analogue scale figures were $6.06 \pm 1.137$ and this is significantly $(\mathrm{p}<0.0001)$ different from that at week $4(3.75 \pm 0.96$, $\mathrm{p}$ $<0.0001)$, at week $8(1.625 \pm 0.67, \mathrm{p}<0.0001)$ and at week $12(0.5 \pm 0.61, \mathrm{p}<0.0001)$.

There was also a slight but not significant weight increase (by $0.48 \% \pm 6.9 \%$, NS) at week 4 compared to week 0 , when administration of ColoPlus was commenced. This weight increase continued to be present at 4 weeks $(+5.11 \% \pm 9.0 \%, \mathrm{p}<0.001)$ and at 8 weeks $(+4.44 \% \pm 7.5 \%, \mathrm{p}<0.0001)$ after stopping intake of ColoPlus.

The immunological capacity as measured by CD4+ counts was increased after 4 weeks of intake of ColoPlus $(+15.5 \% \pm 2.8 \%, \mathrm{p}=0.0001)$, at 8 weeks (i.e. 4 weeks after stopping ColoPlus therapy) by $+39.1 \% \pm 3.9 \%$, $\mathrm{p}<$ 0.0001 . At 12 weeks (i.e. 8 weeks after stopping ColoPlus therapy) it returned to baseline values $(+2.1 \%, \pm 2.8 \%$, NS) (Table 2).

The corresponding figures for serum albumin and hemoglobin showed similar positive effects of ColoPlus and the same time trend (Table 2).

\section{Discussion}

Our results indicate that the colostrum-based food product ColoPlus is effective in boosting immunological capacity in HIV-positive children as measured by the rise in CD4+ counts. There was a concomitant enhancement of blood hemoglobin levels and of serum albumin. These latter figures point towards positive nutritional effects reversing the negative effects of malnutrition. With better nutritional status followed a weight increase and diminished fatigue, thereby improving a sense of well-being and functionality in HIV-positive children.

ColoPlus was well accepted by all children and no intolerance or adverse events were seen.

These findings are consistent with those of previous studies using ColoPlus in adult HIV infected patients [7]-[9].

The children in this field study were both malnourished and HIV infected. This situation tends to occur in impoverished populations and it is well known that malnutrition increases the disease burden of HIV and also lessens the effects of antiretroviral therapy [10]. It is thus of paramount importance that good nutritional care is given to both HIV-positive children and adults.

ColoPlus is thus unique in that it contains colostrum which boosts the immune system by enhancing the pivotal CD4+ levels in HIV-positive patients [7] [8]. ColoPlus also provides good nutritional support [7]-[9].

In this study, and in earlier studies on adults [7] [8], ColoPlus alleviated diarrhea, which can severely diminish quality of life. Chronic diarrhea is also an AIDS defining condition, according to WHO.

In this study, as in earlier studies in adults, ColoPlus had a prolonged effect with a duration of at least 4 weeks after cessation of ColoPlus intake. One can merely specutate on why this happens. One explanation could be that a restoration of the intestinal immune system occurs [9].

The main limitation of the present study is that it is uncontrolled without a placebo-arm. Future randomized placebo-controlled studies will be needed to verify our results.

What is then the role of colostrum-based food products in the treatment of HIV-positive children and adults. The mainstay of therapy of HIV patients is of course ART or HAART. However, in poverty-stricken areas ART or HAART may at present not be at hand and in that case colostrum-based food supplements can have a crucial role in sustaining CD4+ levels and thereby postpone the need for ART or HAART. Also, to ensure optimal effect of ART and HAART colostrum-based remedies may act as an adjunct in the treatment of HIV positive pa- 
tients. However, a randomized controlled trial has been carried out in HIV-1 infected patients with suboptimal CD4+ T cell response to combined ART, by adding hyper immune bovine colostrum. No enhancement of CD4+ $\mathrm{T}$ cell count by colostrum-supplementation was seen [11]. Further studies are though needed to clarify the role of bovine colostrum in the management of patients with HIV infection.

\section{Conclusion}

These results show that addition of a colostrum-based food product to the daily diet is beneficial in HIV-positive malnourished children with a subsequent improvement of nutritional status and immunological capacity. These effects were prolonged and remained at least 4 weeks after cessation of ColoPlus ingestion.

\section{Acknowledgements}

The authors acknowledge the contribution of the IKEA Foundation, Swedish Embassy in Uganda, Ministry of Health-Uganda, the districts of Gulu, Amuru and Nwoya as well as the communities which participated in the study.

\section{Conflict of Interest}

The authors declare that there was no conflict of interest in this research except that Claes-Henrik Florén is on the advisory board of ColoPlus AB, Malmö, Sweden.

\section{References}

[1] Rump, J.A., Arndt, A.A., et al. (1992) Treatment of Diarrhea in Human Immunodeficiency Virus-Infected Patients with Immunoglobulins from Bovine Colostrums. Clinical Investigation, 70, 588-594. http://dx.doi.org/10.1007/BF00184800

[2] Pakkanen, R. and Aalto, J. (1997) Growth Factors and Antimicrobial Factors of Bovine Colostrums. International Dairy Journal, 7, 285-297. http://dx.doi.org/10.1016/S0958-6946(97)00022-8

[3] Plattenberg, A., Stoehr, A., Stellbrink, H.J., Albrecht, H. and Meigel, W. (1993) A Preparation from Bovine Colostrum in the Treatment of HIV-Positive Patients with Chronic Diarrhea. Clinical Investigation, 71, 42-45. http://dx.doi.org/10.1007/BF00210962

[4] Greenberg, P.D. and Cello, J.P. (1996) Treatment of Severe Diarrhea Caused by Cryptosporidium parvum with Oral Bovine Immunoglobulin Concentrate in Patients with AIDS. Journal of Acquired Immune Deficiency Syndromes and Human Retrovirology, 13, 348-354. http://dx.doi.org/10.1097/00042560-199612010-00008

[5] Nord, J., Ma, P., Di John, D., Tzipori, S. and Tacket, C.O. (1990) Treatment with Bovine Hyperimmune Colostrum of Cryptosporidial Diarrhea in AIDS Patients. AIDS, 4, 581-584. http://dx.doi.org/10.1097/00002030-199006000-00015

[6] Beth, L., Ungar, P. and Douglas, J. (1990) Cessation of Cryptosporidium Associated Diarrhea in Acquired Immunodeficiency Syndrome Patient after Treatment with Hyperimmune Bovine Colostrum. Gastroenterology, 98, 486-489.

[7] Floren, C.-H., Chinenye, S., Elfstrand, L., Hagman, C. and Ihse, I. (2006) ColoPlus, a New Product Based on Bovine Colostrum, Alleviates HIV-Associated Diarrhea. Scandinavian Journal of Gastroenterology, 41, 682-686. http://dx.doi.org/10.1080/00365520500380817

[8] Kaducu, F.O., Okia, S.A., Upenytho, G., Elfstrand, L. and Floren, C.-H. (2011) Effects of Bovine Colostrum-Based Food Supplement in the Treatment of HIV-Associated Diarrhea in Uganda. Indian Journal of Gastroenterology, $\mathbf{3 0}$, 270-276. http://dx.doi.org/10.1007/s12664-011-0146-0

[9] Elfstrand, L. and Floren, C.-H. (2010) Management of Chronic Diarrhea in HIV-Infected Patients: Current Treatment Options, Challenges and Future Directions. HIV/AIDS-Research and Palliative Care, 2, 219-224.

[10] Paton, N.I., Sangeetha, S., Earnest, A. and Bellamy, R. (2006) The Impact of Malnutrition on Survival and the CD4 Count Response in HIV-Infected Patients Starting Antiretroviral Therapy. HIV Medication, 7, 323-330.

[11] Byakwaga, H., Kelly, M., Purcell, D.F., French, M.A., Amin, J., Lewin, S.R., Haskelberg, H., Kelleher, A.D., Garsia, R., Boyd, M.A., Cooper, D.A. and Emery, S. (2011) Coral Study Group. Antiretroviral Therapy Intensification with Raltegravir and/or Hyperimmune Bovine Colostrum in HIV-1 Infected Patients with Suboptimal CD4+ T Cell Response: A Randomized Controlled Trial. Journal of Infectious Diseases, 204, 1532-1540.

http://dx.doi.org/10.1093/infdis/jir559 